Revue d'histoire de l'Amérique française

Q REVUE D'HISTOIRE DE L'AMÉRIQUE FRANÇAISE

\title{
Le testament de Samuel Champlain, 17 novembre 1635
}

\section{Robert Le Blant}

Volume 17, numéro 2, septembre 1963

URI : https://id.erudit.org/iderudit/302279ar

DOI : https://doi.org/10.7202/302279ar

Aller au sommaire du numéro

Éditeur(s)

Institut d'histoire de l'Amérique française

ISSN

0035-2357 (imprimé)

1492-1383 (numérique)

Découvrir la revue

Citer ce document

Le Blant, R. (1963). Le testament de Samuel Champlain, 17 novembre 1635.

Revue d'histoire de l'Amérique française, 17(2), 269-286.

https://doi.org/10.7202/302279ar d'utilisation que vous pouvez consulter en ligne.

https://apropos.erudit.org/fr/usagers/politique-dutilisation/ 


\section{DOCUMENTS INÉDITS}

\section{LE TESTAMENT DE SAMUEL CHAMPLAIN}

\section{7 novembre 1635}

\section{HISTOIRE DU DOCUMENT}

Découvert au mois d'août 1959 par Mme Jurgens, conservateur au Minutier central des Archives Nationales, ce curieux document ne manque pas de soulever des problèmes complexes par suite de singularités qui attirèrent l'attention dès qu'il fut soumis à l'examen de juristes. Lors de l'inventaire effectué à partir du 21 novembre 1636 des biens se trouvant en la possession d'Hélène Boullé, veuve du découvreur ${ }^{1}$, dans une maison, à Paris, rue d'Anjou, paroisse Saint-Jean-en-Grève, les prudents notaires parisiens Guillaume Duchesne et Pierre Fieffé s'abstinrent de qualifier un document présenté et représenté, selon leur expression par la veuve consistant en deux feuilles de grand papier dont il n'y avait que deux feuillets et la première page du troisième feuillet écrit, commençant par ces mots: "Au nom du père, du fils et du Saint Esprit, moi, Samuel de Champlain" et finissant par ces mots: "Fait le dix-septième de novembre à Québec mil six cent trente cinq en présence de ceux qui sont ici soussignez, signé en fin Champlain, A. de Brasdefert Chasteaufort, Derré, D. Rousseau, Bouchard, Le Tardif, Giffard, P. Gobbe, une marque allentour (sic) de laquelle est écrit: la marque de Guillaume Couillard, de la Ville, greffier de Quebecq, lequel écrit, ladite damoiselle de Champelain dit lui avoir été envoyé de la Nouvelle-France et luy avoir été apporté le vendredi quatorzième jour du présent mois par le nommé La Treille qui disait venir de la part du sieur Cheffault, étant ledit écrit enveloppé lorsqu'elle le reçut, en un paquet avec les commissions dudit

${ }^{1}$ Archives Nationales, Minutier central, Minutes de Fieffé, LXII, 138, communication de Mme Jurgens, conservateur. 
défunt sieur de Champelain pour le fait de son gouvernement de la Nouvelle-France et après ledit paquet ouvert, elle aurait reconnu que ledit écrit est le testament et ordonnance de dernière volonté dudit défunt sieur de Champelain, son mari, ayant auparavant reçu nouvelles de l'envoi dudit testament par lettre missive du père Lallemant de la compagnie de Jésus et par le sieur Derré à l'instant de laquelle respectivement lecture a esté faite dudit testament par l'un des notaires soussignés, l'autre présent, en la présence desdites parties comparantes aprés laquelle lecture ledit testament a été inventorié sous la cote un et demeuré es mains de Fieffé, l'un des notaires soussignés du consentement desdites parties et elles ce requérant afin d'être gardé par lui pour minute, $\mathrm{y}$ avoir recours et en être délivré expédition à qui il appartiendra aux protestations respectivement faites des parties et mentionnées au procés-verbal dudit sieur commissaire Le Vacher ${ }^{2}$ de la levée dudit scellé, duquel sieur commissaire ledit testament a été paraphé en chacune page d'icelui ne varietur au réquisitoire desdites parties, ledit testament inventorié comme dit est: un."

La rédaction de ce procès-verbal signé par les deux notaires, les parties, c'est à dire Hélène Boullé et Marie Camaret, cousine germaine de Champlain, enfin par noble homme Georges Lefebvre, conseiller du roi, ancien substitut du procureur du roi au Châtelet de Paris, nécessite quelques observations en dépit d'un certain rajeunissement de l'orthographe auquel nous avons procédé.

Le passage "à l'instant de laquelle" est inintelligible si on n'ajoute pas "reconnaissance": c'est seulement à partir de la déclaration de la veuve que les notaires considèrent la pièce comme un testament. Leur prudence ne fut pas exempte de quelqu'inattention, car ils changèrent sans raison apparente l'ordre d'énumération des témoins dont la liste doit être rétablie ainsi d'après les signatures et la marque suivies horizontalement en adoptant provisoirement leur lecture: A de Bras de Fer Chateaufort, Derré, D. Rousseau, Bouchard, Giffard, Le Tardif, P. Gobbe, Guillaume Couillard, de la Ville, greffier.

2 Document perdu. 
Il faut en retenir que le document se trouva d'abord entre les mains du père Charles Lallemant, de la Cie de Jésus et de François Derré, dit Gan, membre de la Cie des Cent-Associés veillant sur ses intérêts en Nouvelle-France, qu'il franchit l'océan dans des conditions ignorées pour parvenir à Antoine Cheffault, avocat au parlement de Paris, un des dirigeants les plus actifs à l'époque de la Cie des Cent-Associés, puis, par l'intermédiaire d'un certain La Treille, personnage inconnu de nous à la veuve qui prit l'initiative de le considérer comme le testament de Champlain, reconnaissant ainsi au moins implicitement l'authenticité d'une signature évidemment très déformée.

Cette attitude d'Hélène Boullé semble au premier abord avoir réduit certaines difficultés car les dispositions prises lui étaient peu favorables, la privant par toute une série de legs de tout ce que le défunt possédait au Canada contrairement à ses droits d'usufruit, mais cette reconnaissance pouvait être opposée à Marie Camaret, héritière du défunt, particulièrement touchée sur le terrain de la nu-propriété et les problèmes à résoudre s'en trouvèrent compliqués.

\section{DESCRIPTION DU TESTAMENT}

Le texte est rédigé d'une bonne écriture, petite, mais très lisible, apprise au XVI $\mathrm{XI}^{\mathrm{e}}$ siècle, comportant certaines ornementations, notamment de nombreuses majuscules. On n'y trouve aucune ressemblance avec l'écriture de Champlain que nous connaissons par une inscription apposée sur une carte de la Nouvelle-France ${ }^{3}$ comme grande, simple et cursive. La grande élégance du style concorde avec la pénurie des fautes d'orthographe constituée surtout par de fréquentes omissions des " $\mathrm{s}$ " qui auraient été utiles pour caractériser les pluriels, péché véritablement véniel à l'époque, et nous sommes loin du jargon obscur des passages de la publication imprimée des « Voyages de Champlain » qui sont susceptibles d'être attribués à la plume du découvreur. ${ }^{4}$

${ }^{3}$ Carte de 1607 reproduite par Biggar dans les pièces considérées comme des annexes de sa publication: The works of Samuel Champlain (Toronto, 1928) $8^{\circ} \mathrm{Pa} 78$ bis, à la Bibliothèque Nationale.

4 Voir notre article en collaboration avec M. Delafosse: "Les Rochelais dans le Saint-Laurent", Revue d'Histoire de l'Amérique française, X (décembre 1956) : 341. 
Il apparaît immédiatement que ce texte n'est pas l'œuvre d'un juriste, car un renvoi en marge, constituant une disposition importante, n'est pas approuvé. Une telle méconnaissance des données pratiques les plus élémentaires n'empêcherait pas a priori de penser qu'on se trouve en présence de l'œuvre d'un certain de Laville qui signa au bas de l'acte en prenant la qualité de greffier de Québec. Il n'y avait alors dans la future capitale ni notaires, ni organisme de juridiction permanent, les conseils de guerre étant seulement susceptibles d'être réunis pour une cause déterminée ainsi qu'on l'avait fait lorsqu'il avait fallu réprimer une révolte, lors de la fondation de Québec, en $1608 .{ }^{5}$ La qualité de greffier semble donc bien avoir été prise ici seulement pour les besoins d'une cause autre que l'établissement d'un acte sincère.

$\mathrm{Au}$ demeurant, il suffit de comparer l'écriture du testament avec celle de Laville dont nous avons deux échantillons pour que son intervention puisse être écartée. L'ornementation de sa signature suffirait à justifier ses qualités d'écrivain manifestées aussi dans l'inscription "la merc de Guillaume Couillard". Son écriture est plus petite que celle du testament avec un caractère personnel très différent, les lettres étant presque toujours reliées entre elles, ce qui est l'exception dans le corps du testament où l'on trouve en outre l'orthographe Kebec, alors que Laville a écrit "Quebec". On peut donc écarter l'hypothèse d'une rédaction du prétendu greffier, le document pouvant avoir été écrit par au moins quatre autres témoins dont la signature est habilement ornementée: Brasdefer-Chateaufort, Giffard, Derré et Letardif.

Le testament commence par un préambule comprenant d'abord, selon les usages de l'époque, une déclaration par laquelle son auteur expose qu'il est sain d'esprit et ne veut pas être surpris par la mort sans avoir déclaré ses dernières volontés. Ce préambule continue sous la forme d'une profession de foi catholique et de charité chrétienne également admissible en dépit de certaines particularités de style et d'une reconnaissance de propriété des biens du testateur en faveur de Dieu. Cette

5 Biggar, op. cit., II : 25 et suiv. 
abnégation se concilie mal, bien que nous y conduisant, avec la première disposition testamentaire qui est aussi la plus originale.

La Sainte Vierge est instituée héritière des meubles, de l'or et de l'argent possédés au Canada par Champlain. Le rédacteur marqua aussitôt une hésitation en identifiant la première légataire avec la chapelle de Notre-Dame-de-Recouvrance qui lui avait été dédiée. C'est auprès de cet immeuble qu'il sollicita la permission de limiter son premier legs par d'autres immédiatement énumérés, priant $M$. Gan de les exécuter et de faire remettre ses papiers au père Lallemant.

Le sort des biens de France fut ensuite réglé très simplement comme revenant à Hélène Boullé en exécution d'une donation réciproque. Des complications ne paraissaient pouvoir intervenir que si le testateur décédait veuf, Marie Camaret, légataire universelle, se trouvant alors à devoir régler des legs particuliers nombreux et importants. M. Sirou et à son défaut sa femme furent chargés d'exécuter le testament pour ces biens se trouvant en France.

La signature de Champlain est malhabile et très déformée si on la compare à celles qu'il donna antérieurement. Les huit témoins dont les signatures précèdent celle du greffier, le numéro d'inventaire et un procès-verbal des notaires doivent être énumérés ainsi d'après la paléographie et la disposition des seings de gauche à droite: A de Bras de fer chateaufort, Derré, D Rousseau, Boullard, Giffard, Letardif, P Goblet, Guillaume Couillard. Le testament donne sur la façon dont s'habillait le découvreur des indications précieuses qui ne nécessitent pas de développements, mais l'identification des personnages mis en cause par le document peut apporter des éclaircissements. Nous les examinerons successivement suivant l'importance de leur intervention.

\section{LES HOMMES DE CONFIANCE DE CHAMPLAIN}

Le père Charles Lallemant est un personnage bien connu, mais au sujet duquel certaines précisions pourraient encore être apportées. Portant d'argent à un chevron d'azur chargé de trois étoiles d'argent au chef de gueules à trois étoiles d'or, fils de 
Gabriel Lallemant et de Madeleine d'Auvergne, il était l'arrièrepetit-fils de François Lallemant, avocat au parlement, le petitfils de Simon Lallemant, sieur de Pierrefitte et d'Étiennette Sanguin, elle-même fille de Claude Sanguin, bailli du Louvre, et d'Étiennette Lescuyer. Le père Charles Lallemant naquit probablement dans une maison située rue de La Poterie, paroisse Saint-Merry, où son père demeurait dès le 12 février $1583 .^{\circ}$ Honorable homme, maître Gabriel Lallemant, conseiller aux Eaux et Forêts dès le 4 janvier 1580, avait épousé, suivant contrat du 22 septembre 1579,7 Madeleine d'Auvergne, fille de François d'Auvergne, seigneur de Dampont, conseiller en la Chambre du Trésor et de Madeleine Guibert. Elle mourut avant le 26 août 1609, date de l'inventaire de ses biens, effectué dans la maison de la rue de La Poterie, comprenant une bibliothèque importante. Devenu lieutenant criminel des ville, prévôté et vicomté de Paris, Gabriel Lallemant n'eut pas d'enfant d'un second mariage passé suivant contrat du 8 novembre 1613 avec Élisabeth Griffon, fille de Jean Griffon, écuyer, sieur de Villebouzin, et de damoiselle Henriette de Gaumont. ${ }^{8}$ Outre son frère aîné, Pierre et son frère cadet Jérôme, tous deux jésuites comme lui, Charles Lallemant eut un autre frère, Christophe, secrétaire du roi et au moins deux sœurs: Lyée et Anne. Lyée Lallemant épousa Jacques Lallemant, avocat au parlement et Anne se maria avec Guillaume du Fayot, secrétaire du roi, seigneur de Cuisy. Elle était la mère de Jean du Fayot, qui figura au $\mathrm{N}^{\circ} 120$ de la liste des Cent-Associés de la Cie de la Nouvelle-France rectifiée par nous et demeurait encore rue de La Poterie, le 5 mai 1654, date à laquelle elle gratifia son fils Jean, de la terre et seigneurie de Cuisy, près de Dammartin. ${ }^{9}$

Le père Charles Lallemant était, en outre, cousin germain d'Arnould Lallemant, fils de Jacques Lallemant, sieur de Pierrefitte et d'Anne Morelli, fille de noble homme Augustin Morelli

\footnotetext{
${ }^{6}$ Pièces originales 1624 , article Lallemant, citant une note de Blanchard, Catalogue des conseillers au parlement, Fonds français 32515 , fo. 5 , l'Allemand.

7 Inventaire des biens de Madeleine d'Auvergne, du 26 août 1609, minutes de Simon Fournier, III : 487.

8 Idem, III: 495.

9 Cf. y 188 , fo. 83, vo., aux Archives Nationales.
} 
et d'Anne de Tavanne, sœur de Catherine Morelli, femme de noble homme Charles Tristan, auditeur en la Chambre des Comptes et de Marie Morelli, alias Morely, épouse de Gilles de Maupeou, sieur d'Ablèges, conseiller d'État, intendant des Finances, le 22 décembre $1616 .{ }^{10}$ Bien que figurant correctement au $\mathrm{N}^{\circ} 12$ de la liste des Cent-Associés de la Cie de la NouvelleFrance rectifiée par nous, le nom de François Derré, dit le sieur Gan, alias Gand, a subi des déformations presqu'incroyables au cours des notices qui lui ont été consacrées par Dionne ${ }^{11}$ et dans le Bulletin des Recherches Historiques. ${ }^{12}$ On le trouve à Paris, le 12 janvier 1618, demeurant rue de Jouy, chez un maître des requêtes dont il était le secrétaire ${ }^{12 b i s}$ et premier commis de la Cie au Canada, dès le 22 juillet 1628, date à laquelle il devait faire charger du poisson dans le navire La Jeanne, de Bordeaux, commandé par Jean de Nioche. ${ }^{13}$ Bénéficiant d'une considération tout à fait particulière de la part du découvreur, Aymé Sirou n'était connu, jusqu'à présent, que par son inscription au $\mathrm{N}^{\circ} 71$ de la liste des Cent-Associés, avec les qualités de conseiller du roi, trésorier général de France à Paris. On le trouve déjà secrétaire du roi, logeant en la capitale, rue Tirechappe, paroisse Saint-Germain-l'Auxerrois, lorsqu'il donna une procuration devant Contesse, notaire au Châtelet, ${ }^{14}$ le 23 juillet 1599. Trésorier général de la marine du Levant, demeurant rue Sainte-Croixde-la-Bretonnerie, paroisse Saint-Germain-en-Grève, il régla, le 17 février 1611, avec Jacob Girard, sieur d'Espinais, les gages du frère de ce dernier, capitaine entretenu en la marine, ${ }^{15}$ puis, vendit, le 18 mai suivant, d'après un factum de 1623, à Ithier Hobier, trésorier général de France à Bourges, figurant au $\mathrm{N}^{\circ} 40$ de la liste des Cent-Associés, son office de trésorier général de la marine du Levant, fortifications et morte paye de Provence. ${ }^{16}$

${ }_{10}$ Partage de ce jour, minutes de Haultdessens, LIV : 317.

11 Samuel Champlain (Québec, 1891), II : 461, $8^{\circ} \operatorname{Ln}^{27} 42429$, à la Bibliothèque Nationale.

12 Table à la disposition des lecteurs à la salle de travail, idem. 12bis Minutes de Richer, LI: 123.

13 Minutes de François Duboys, 3 E 4871, fo. 727, aux Archives départementales de la Gironde.

14 Minutes de Contesse, LIV : 237, aux Archives Nationales.

15 Registre de Bigre, VI: 177, idem.

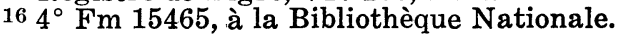


Il épousa, avant le 6 mars 1623, Elisabeth Le Sergent, fille de Me Claude Le Sergent, sieur de Faronville, et de dame Charlotte Chahu. ${ }^{17}$ Elle avait une sœur, Suzanne, qui épousa Thomas Leclerc, conseiller d'État, secrétaire du cabinet du roi, surintendant, commissaire général des armées et munitions du roi, le 7 janvier $1620 .{ }^{18}$ Son père mourut conseiller correcteur à la Chambre des Comptes avant le 16 décembre 1616, date à laquelle Charlotte Chahu demeurait rue du Cocq, paroisse Saint-Eustache. ${ }^{19}$ Aymé Sirou était trésorier général de la généralité d'Orléans et demeurait avec sa femme, au marais du Temple, rue d'Anjou, le 6 mars 1623, lorsque tous deux contractèrent un emprunt auprès de Barbe Dolu, veuve de Jacques Vallée, sieur des Barreaux. ${ }^{20}$ L'obligation fut solidaire avec Ithier Hobier, demeurant alors rue Sainte-Avoye, paroisse Saint-Merri, marié avec Geneviève Bergeon et plusieurs autres futurs associés de la Cie de la Nouvelle-France: noble homme Robert Godefroy, receveur général des finances à Tours, marié avec demoiselle Marie Marteau ( $\mathrm{N}^{\circ} 53$ ), noble homme Pierre Robineau, aussi receveur général des finances à Tours, demeurant rue de Berry, paroisse Saint-Nicolas-des-Champs, marié avec demoiselle Renée Marteau ( $\left.\mathrm{N}^{\circ} 42\right)$ et noble homme Jacques Bordier, secrétaire du roi, ancien receveur général des gabelles du Lyonnais, demeurant rue du Plastre, paroisse Saint-Jean, marié avec dame Catherine Lybault $\left(\mathrm{N}^{\circ} 55\right)$. Aymé Sirou demeurait rue Diane, clôture Sainte-Catherine, paroisse Saint-Germain, le 29 juillet 1632, lorsque, devant Bruneau, notaire au Châtelet, il fit donation $\mathrm{du}$ quart de l'office de greffier héréditaire alternatif de Villefranche-de-Rouergue au Collège des jésuites de Clermont, rue Saint-Jacques, à Paris, pour la réception de son fils Claude. ${ }^{21}$ Propriétaire d'un office dans l'élection de Bayeux en $1634,{ }^{22}$ Sirou mourut après le 21 février 1637 et avant le 14 mai 1638. A cette date, Elisabeth Le Sergent, intitulée veuve et tutrice de leurs enfants mineurs, demeurait rue des Prestres, paroisse

17 Acte du 23 mai 1635, minutes de Mottelet, XC: 197.

18 Minutes de Contenot, XVI: 208.

19 Minutes de Le Voyer, XII: 45.

20 Minutes de Blosse, LXXV: 2.

21 y 173, fo. 146, d'après les minutes de Bruneau, XC: 37.

22 Pièces originales 2710, art. Sirou, à la Bibliothèque Nationale. 
Saint-Paul, lorsqu'elle accorda une pension de 600 livres, ${ }^{23}$ à son beau-frère, Jean Sirou, écuyer, sieur de La Salle, demeurant au marais du Temple, rue de Poitou, paroisse Saint-Gervais, à qui son mari avait constitué une rente devant le même notaire, le 21 février 1637. Elle soutenait, le 25 janvier 1640, contre Claude de Bragelonne, associé de la Cie de la Nouvelle-France, sous le $\mathrm{N}^{\circ} 54$, un procès ${ }^{24}$ qui durait encore le 1er février suivant. ${ }^{25}$ Elle se trouvait au couvent des Carmélites d'Orléans, le 29 avril 1650, lorsqu'elle fit une donation à son fils, Claude Sirou, seigneur de Marbonne, conseiller et aumônier du roi, demeurant rue de la Cerisaie, paroisse Saint-Paul. ${ }^{26}$

Le révérend père Alexandre Jarry passa, le 7 septembre 1640 , comme procureur de la maison professe des Pères jésuites de Paris, un acte avec Euphase de Saint-Pierre, procureur des carmes des Billettes. ${ }^{27}$

\section{LES PERSONNES PHYSIQUES LÉGATAIRES DE BIENS SE TROUVANT AU CANADA}

Les identifications ne sont pas facilitées par l'absence d'une publication intégrale des plus anciens actes figurant sur les registres paroissiaux de Québec.

Marin, maçon, demeurant vers la maison des Pères récollets peut avoir été Marin Boucher. ${ }^{28}$ Le valet Poisson et Bonaventure, filleul de Champlain, restent à rechercher. Hélène Boullé, femme du découvreur, mérite une étude particulière.

Hélène, femme de M. Hébert, est Hélène Desportes, native de Québec, fille de Pierre Desportes et de Françoise Langlois qui avait épousé, le 1er octobre 1634, Guillaume Hébert, fils de Louis. ${ }^{29}$ Marguerite, filleule de la femme de Pivert, se traduit par Marguerite Martin, née à Québec, le 4 janvier 1624, et baptisée le 16. Elle eut effectivement comme marraine Margue-

23 Minutes de Galloys, LXXV: 37, aux Archives Nationales.

$24 \mathrm{X}^{\mathrm{IB}} 5445$, idem.

25 y 187 , fo. 238 , d'après un notaire d'Orléans.

26 y 187, fo. 238 , d'après un notaire d'Orléans.

27 Minutes de Richer, LI: 197.

28 Recensement de 1635 publié par Dionne, op. cit., II : 458.

29 Communication du regretté R.P. Archange Godbout, o.f.m. 
rite Lesage, femme de Nicolas Pivert. ${ }^{30}$ Hélène, cousine de Marguerite Martin est Hélène Desportes que nous connaissons. Madame Giffard s'identifie facilement avec Marie Regnouard, née à Mortagne, le 8 décembre 1599, mariée suivant contrat du 11 février 1628 avec Robert Giffard que nous trouverons, comme témoin. ${ }^{31}$ Le père Charles Lallemant a été étudié précédemment.

\section{LES PERSONNES PHYSIQUES EVENTUELLEMENT LÉGATAIRES DES BIENS SE TROUVANT EN FRANCE}

Abraham et sa femme sont Abraham Martin, dit l'Écossais, et sa femme, Marie Langlois. Leur fille Marguerite avait déjà bénéficié d'un legs sur les biens du Canada. Sa sœur Hélène fut effectivement la filleule de Champlain. Hélène Desportes apparaît une fois encore. Marguerite Couillard fut bien la filleule de Champlain. ${ }^{32}$

Pyvert et Nicolas Pivert, considéré comme arrivé au Canada dès $1613 .^{33}$ La femme de La Caille est Simone Orville, alias d'Orgeville, épouse d'Adrien d'Abancourt, dit La Caille, originaire de la paroisse Saint-Waast, de Soissons. ${ }^{34}$

\section{LES PERSONNES MORALES ÉVENTUELLEMENT LEGATAIRES DES BIENS SE TROUVANT EN FRANCE}

La mission des Pères jésuites à Québec fut la mieux servie, recevant les parts du testateur dans la Cie de la Nouvelle-France et dans la Cie particulière du fleuve Saint-Laurent, acquises pour 3,000 et 900 livres, plus 400 livres, soit, en tout 4,300 livres. La distribution s'étendit, ensuite, en faveur d'organismes parisiens charitables ou pieux qui reçurent, l'hôpital de la Charité, faubourg Saint-Germain: 400 livres; les Hospitalières, près des Minimes de la Place Royale: 400 livres; les pères de la Doctrine chrétienne à Paris: 600 livres; les pauvres du Grand-Hôpital:

30 I dem.

31 Nova-Francia, IV: 324 ; Bulletin des Recherches Historiques (septembre 1903) : 267-270.

32 Ferland, Notes sur les registres de Québec (Québec, 1863), Lk ${ }^{12}$ 933, à la Bibliothèque Nationale.

33 Dionne, op. cit., II : 423.

34 R.P. Archange Godbout, "Nos ancêtres au 17e siècle", Rapport de l'Archiviste de Québec, pour 1951-1952, 1952-1958, 459. 
400 livres; les pauvres honteux: 600 livres; l'église du SaintEsprit, vers la Grève: 100 livres; les pères Minimes de la Place Royale: 500 livres.

\section{LA LEGGATAIRE UNIVERSELLE}

Légataire de ce qui resterait, la cousine de Champlain, fille du feu capitaine Georges Camaret, maintenant mariée au sieur d'Arsant, demeurant à La Rochelle, fit l'objet d'une précision inédite concernant l'identité de son père, tandis que le nom de son époux était étrangement déformé. Née vers 1592, comme âgée de plus de 28 ans, le 2 mars 1620, fille héritière de Françoise Le Roy, tante maternelle du découvreur qui avait été son tuteur, ${ }^{35}$ elle avait épousé, avant le 15 mars 1619, Jacques Hersant, alors piqueur des chiens de la chambre du roi, demeurant à La Celle en Brie, ${ }^{36}$ au lieu dit Courbon en Brie, paroisse de Guérart. ${ }^{37}$ Encore piqueur des chiens de la chambre du roi, le 2 mars 1620, Jacques Hersant devint conseiller des Traites foraines et domaniales de La Rochelle, puis contrôleur des Gardes de ce bureau, habitant effectivement à La Rochelle, où il passa une location, le 9 octobre $1636^{38}$ et une sous-location, en $1639 .{ }^{39}$ Il devint associé de la Cie de la Nouvelle-France, sous le $\mathrm{N}^{\circ} 132$, par suite de la cession faite, le 5 septembre 1639, par Hélène Boullé, des parts ayant appartenu au découvreur ${ }^{40}$ et, de son mariage avec Marie Camaret, naquit au moins un fils, Claude Hersant, ${ }^{41}$ qui passa un acte avec elle, le 13 juillet 1637.

\section{LES TÉMOINS}

Antoine de Bras-de-Fer de Châteaufort, signalé comme un chevalier de Malte ayant pris le commandement après la mort

\footnotetext{
${ }^{35}$ Arrêt du parlement de Paris, du 20 août 1639, $\mathrm{X}^{\mathrm{IB}}$ 177, aux Archives Nationales.

36 Revue d'Histoire des Colonies, XXXVII (1950) : 203.

37 Vente du 2 mars 1620, minutes de Caron, XXIII : 258, aux Archives Nationales.

38 Registre de Teuleron de 1636, fo. 66, vo., aux Archives de la Charente maritime.

39 Idem, de 1639-40, fo. 47.

40 Minutes de Fieffé, LXII : 144, aux Archives Nationales.

41 Minutes de Gaultier, IX: 379, idem.
} 
de Champlain, ${ }^{42}$ peut avoir été Marc-Antoine de Bras-de-Fer, fils de Jean, seigneur de Chateaufort et d'Anne Gillot. ${ }^{42 b 1 s}$ Nous avons déjà étudié François Derré, dit le sieur Gan. Quant à D. Rousseau, n'étant pas possible de l'identifier avec Pierre Rousseau qui signa la protestation d'Émery de Caen du 22 août 1631,43 il peut seulement s'agir d'un Rousseau indiqué sans prénom dans le recensement de $1635 .{ }^{44}$ Bouchard paraîtrait, a priori, représenter Théodore Bochart, sieur Duplessis, général de la flotte de la Nouvelle-France, mais l'examen paléographique de sa signature ne permet pas de supprimer l' " $u$ " et donne la lecture "Boullard". Nous restons donc en présence d'un inconnu de nous. Robert Giffard, anobli par lettres du mois de mars 1658 a fait l'objet de publications suffisantes. ${ }^{45}$ Olivier Le Tardif, sieur de La Porte, commis de Guillaume de Caen, ${ }^{46}$ puis de la Cie des Cent-Associés, ${ }^{47}$ épousa Louise Couillard, fille de Guillaume, puis Barbe Aymard, originaire du Poitou. P. Gobb dont la signature se lit "P.Goblet" est encore un inconnu. Guillaume Couillard semblerait avoir été originaire de la paroisse Saint-Landry à Paris, comme fils d'autre Guillaume Couillard et d'Élisabeth de Vesins, ${ }^{48}$ né en 1591.

Le greffier de Laville paraît avoir rédigé, le 23 décembre 1635, la mise en possession de Guillaume Huboust, par François Derré, d'une terre voisine de celle de Guillaume Couillard. Il s'agit, vraisemblablement, de Jacques de Laville, caporal au fort de Québec, témoin au contrat de mariage de Nicolas Macart, avec Marguerite Couillard, veuve de Jean Nicolet, reçu par le notaire canadien Beaucheron, le 12 octobre 1646.49

\footnotetext{
42 "Relation du Père Lejeune", The Jesuit Relations, IX: 206, Lk12 1464 , à la Bibliothèque Nationale.

42 bis Cf. Richer, LI: 120, Inventaire du 24 II 1616.

43 Dionne, op. cit., II : 532, d'après State Paper Office, VI : 23.

44 Dionne, op. cit., II: 465.

45 Bulletin des Recherches Historiques (septembre 1903) : 267-270.

46 Arrêt du Parlement de Rouen, statuant en matière civile, du dernier octobre 1633, registre de septembre-décembre 1633, aux Archives de la Seine Maritime.

47 Bulletin des Recherches Historiques, XX: 24.

48 Abbé Couillard-Després, La première famille française au Canada, $87,8^{\circ} \mathrm{Pz} 1307$, ̀̀ la Bibliothèque Nationale.

49 P.-G. Roy, "Les notaires sous le régime français", Rapport de $l^{\prime}$ Archiviste de Québec, 1922, 4, $4^{\circ} \mathrm{Nt} 3657$, à la Bibliothèque Nationale.
} 


\section{L'INTERPRETATION DU TESTAMENT}

L'exécution portant sur les biens se trouvant au Canada ne semble pas avoir rencontré de difficulté, car l'avocat Boileau paraissant, avec raison, avoir identifié plus tard ${ }^{50}$ la Sainte Vierge et la chapelle de Notre-Dame-de-la-Recouvrance avec les révérends Pères jésuites, ceux-ci ne réclamèrent que les legs portant sur les biens situés en France. Il est infiniment probable qu'Hélène Boullé fut privée de ses droits de partage et d'usufruit sur les biens laissés par le découvreur au Canada.

En ce qui concerne le sort des biens situés en France, il semble, à notre humble avis, que leur dévolution n'aurait dû faire l'objet d'aucune contestation, puisque les legs étaient soumis à la condition non réalisée d'un prédécès d'Hélène Boullé. Cette solution simple ne fut pas mise assez en évidence à l'époque parce que le rédacteur du testament avait signalé l'existence, au profit de la veuve, d'une donation en propriété purement imaginaire, puisque, par contrat de mariage et donation mutuelle, Champlain avait stipulé seulement une donation d'usufruit. Les juristes du XVIIe siècle rétablirent la situation de fait en la compliquant $d u$ point de vue juridique au lieu d'interpréter strictement la volonté du défunt, d'après le texte. Il est certain que Champlain devait savoir ce qu'il avait donné à sa femme et on peut se demander si la suggestion qui fut envisagée, étant donnée l'importance des legs, fut complète. Peut-être faut-il discerner ici une défense des intérêts de son héritière par le découvreur qui persuada l'existence d'une donation en propriété à un révérend père dont les directives auraient trouvé un fondement en cas de survie d'Hélène Boullé, s'il n'avait rencontré qu'une mention sincère de donation d'usufruit, lors de la discussion du testament. Un tel mensonge peut sembler tantôt subtile, tantôt malhabile, mais l'importance qui lui fut accordée lors du procès tend à démontrer que Samuel Champlain sut réussir dans une ultime résistance.

33, rue de Turin,

(Paris (8).

ROBERT LE BLANT

${ }^{50}$ Revue d'Histoire des Colonies (1950) : 224. 


\section{PIËCE JUSTIFICATIVE}

Au nom du Pere et du Filz et du Saint Esprit. Moy, Samuel de Champlain, sain d'esprit et d'entendement, considerant qu'il n'y a rien de si incertain que l'heure de la mort, ne desirant pas estre surpris sans declarer mes dernieres volontez, je laisse ce present escrit afin qu'elles soient manifestes et notoires a tout le monde.

Donc, mon Dieu, constitué en vostre presence et de toute vostre cour celestre, je proteste que je veux vivre et mourir en la foy et religion catholique, apostolique et romaine et recevoir tous les sacremens dont je puis estre capable qu'elle ne refuse point a ses enfans. Je suis prest de signer de mon sang et de ma mort toutes les veritez qu'elle propose a croire comme aussy l'obeissance a vos saincts commandemens.

Je pardonne de bon coeur a tous ceux qui m'ont offencé et ce pour l'amour de vous, ô mon Dieu qui le voulez et le desirez ainsi et supplie bien humblement tous ceux que j'ay offencé me faire ce bien que de me pardonner

Vous m'avez donné une ame raisonnable, ô mon Dieu, je la remetz entre vos mains vous suppliant d'en disposer pour vostre gloire. Pour mon corps, je le laisse icy en depost jusques a la Resurrection derniere que vous le reunirez a mon ame pour participer à sa beatitude.

Scachant en outre que je ne suis que le despensier de ce que vous m'avez mis en main, voicy comme je dispose des biens temporels que vous m'avez donné. Je désire donc, ô mon Dieu, que la tres saincte Vierge vostre mere soit heritiere de ce que j'ay icy de meuble, d'or et d'argent. Je donne donc à la chapelle de ce lieu dediée en son nom et appellée vulgairement Nostre-Dame de Recouvrance tout ce qui se trouvera icy m'appartenir fors et exepté ce qui s'ensuit dont je luy demande permission de disposer en faveur de quelques personnes

Donc, avec sa permission je donne à Marin * macon, demeurant vers la maison des Peres Recollects le

* Espace blanc dans le manuscrit. 
dernier habit que j'ay faict faire de l'estoffe que j'ay pris au magazin.

A Poisson, mon valet, outre l'habit que je luy ay baillé qui n'est pas encore achevé, je luy donne un hault de chausse de bure avec une camisole de petite sergette grise et rouge

Je donne à Bonaventure, mon filleuil, un habit de drap d'Angleterre, pourpoinct, hault et bas de chausse de mesme couleur

Je prie le Pere Charles Lalemant d'envoier à ma femme les Agnus Dei que j'ai avec une peau de renart grise et deux peaux de loutre et une bague d'or dans le chaton de laquelle il y a une espece de diamant

Je donne à Helene, femme de monsieur Hebert, une paire de brassiere de futaine blanche.

J'en donne une autre de mesme estoffe a Marguerite, filleule de la femme de Pyvert avec des espingles et esguillettes de soye qu'elle partagera esgallement avec sa cousine Heleyne.

Je donne a Madame Giffard le tableau de NostreDame qui est dans ma chambre.

Je donne au Pere Charles Lalemant le tableau de Nostre-Seigneur crucifié qui y est aussy et ma boussole et l'astrolabbe de cuivre avec le compas de proportion le suppliant de se saisir de tous mes papiers afin de les faire tenir à ma femme et à cet effect je prie Monsieur Gan (qui me fera s'il luy plaist ce bien de tenir la main à ce que tout ce que dessus soict executé) je le prie dis-je de faire en sorte que lesdictz papiers soient mis es mains au dict Pere.

Maintenant, pour le reste de mes biens que j'ay en France, si mon decez arrive auparavant celuy de ma femme, je n'en puis disposer luy en ayant faict une donation totale au cas qu'elle vint a survivre apres moy, comme aussy m'a elle faict une donation de son bien au cas que sa mort arrivast auparavant la mienne. Et partant si à l'heure que je faictz ce testament ma femme est décédée voicy comme je dispose de tout le bien que j'ay et en France.

Je donne a la Mission des Peres de la compagnie de Jesus à Kebec les trois mille livres que j'ay en la 
Compagnie générale et neuf cents livres que j'ay en la Compagnie particuliere, desirant qu'ils en emploient cinq cent livres pour orner la chapelle de Nostre-Dame de Recouvrance située à Kebec, de tapisseries ou autres meubles servans a l'autel. Et outre ce, je donne à ladicte mission quatre cent livres me recommandans aux prieres des Pères et freres qui sont icy et les suppliant pour la bonne affection qu'ils me portent de dire tous les ans au jour de mon decez une messe pour le repos de mon ame.

Je donne à Abraham et à sa femme six cent livres à la charge qu'ils les emploieront a desfricher des terres en ce pais de la Nouvelle France pour leurs necessitez.

Je donne à Marguerite, fille d'Abraham, six cent livres pour l'aider à se marier en ce pais de la Nouvelle France à un homme qui sera resident en ce dict pais et non autrement et à Hélene son autre fille, ma filleule, trois cent francs.

Je donne trois cent livres à Helene des Portes, filleule de ma femme.

Je donne trois cent livres à Marguerite Couillart, ma filleule, pour aider à la marier.

Je donne à Pyvert deux cent francs.

Je donne cent livres à la femme de La Caille.

Je donne à l'hospital de la Charité situé au fauxbourg Sainct-Germain à Paris quatre cent livres.

Je donne aux Hospitaliéres de Paris logées proche des Minimes de la place Royale quatre cent livres. Je donne six cent livres aux Peres de la Doctrine Chrestienne de Paris à condition qu'ils priront Dieu pour moy et diront quelques messes pour le repos de mon ame.

Je donne quatre cent livres aux pauvres du grand hospital de Paris.

Je donne six cent livres aux pauvres honteux qui ne subsistent que par les charitez des personnes qui scavent leurs nécessitez desirant que les dictes six cent livres soient mises entre les mains de monsieur Sirou ou de madamoiselle sa femme pour les distribuer suivant mon intention et à leur default je desire qu'elles soient mises entre les mains du Pere Jarry 
de la Compagnie de Jesus pour les distribuer à mestre fin.

Je donne cent livres à l'eglise du Sainct-Esprit vers la Greve à condition qu'on y dise trente messes pour moy.

Je donne cinc cent livres aux Peres Minimes de la place Royale de Paris à condition qu'ils disent cent messes pour le repos de mon ame.

Tout le reste de ce qui me peut appartenir tant en meubles qu'immeubles, j'ordonne qu'il soit donné à une mienne cousine, fille du feu capitaine George Camaret maintenant mariée au sieur d'Arsant demeurant à la Rochelle.

Je nomme pour executeur de ce mien testament touchant le bien que j'ay en France ** monsieur Sirou le priant tres humblement pour l'affection qu'il me porte d'en vouloir prendre la peine et l'en prie d'autant plus volontiers que je scay qu'il n'y aura pas grande difficulté en l'execution d'iceluy.

Faict ce dixseptiesme de novembre à Kebec mille six cent trente cinq en presence de ceux qui sont icy soubsignez

Champlain

A.de Bras de fer

Chateaufort

D Rousseau

Boullard

Giffard

P. Goblet
Derré

Le Tardif

le merc de Guillaume ***

Couillard

De Laville

greffier de Quebec

enregistré ung

Ce jourd'huy vingt deuxiesme novembre mil six cens trente six, du consentement et au requisitoire de damoiselle Heleyne Boullé vefve de feu messire Samuel de Champelain, vivant sieur dudit lieu et de damoiselle Marie Camaret, femme et procuratrice de sieur Jacques Hersant et de noble homme Georges

** Le texte est en marge depuis "touchant le bien".

*** La marque de Guillaume Couillard est constituée par une croix. placée à gauche d'un cercle orné de deux traits partant vers la droite en haut et en bas avec inclinaison vers le haut et le bas du document. 
Le Febvre, conseiller du Roy et substitud au Roy de monsieur le procureur de Sa Majesté au Chatelet de Paris et conformement à ce qui est porté par l'inventaire encommencé le jour d'hier par les notaires gardesnottes soubzsignez à la requeste et presence desdites partyes es noms et qualitez y declarez des biens demourez apres le decedz dudit deffunct sieur de Champlain, le present testament a esté delaissé à Fieffé l'un desdits notaires affin d'estre par luy gardé pour minutte, y avoir recours et en estre delivré des expeditions à qui il appartiendra aux protestations respectivement faictes par les partyes y mentionnées au proces-verbal de maistre Claude Le Vacher, commissaire et examinateur au Chatelet pour la levée du scellé qui avoit esté apposé sur lesdits biens et ont signé

Helene Boullé, Marie Camaret, Le Febvre, Duchesne, Fieffé."

**** Archives Nationales, Minutes de Fieffé, LXII: 138, communication et transcription de Mme Jurgens, conservateur. 